\title{
Review on the Role of Flexibility in Iranian's Traditional Housing, Case Study: H. H. Ghafouri's (Ghazviniha) Building
}

\author{
Mehri Eshaghi, ${ }^{1,}$, Gholamreza Bahreini' ${ }^{2}$ \\ ${ }^{1}$ Department of Architecture, Shahrekord Azad University, Shahrekord, Iran \\ ${ }^{2}$ Department of Civil Engineering, University Of British Colombia, Vancouver, Canada
}

Email address:

mehri.eshaghi@yahoo.com (M. Eshraghi), bahreini@alumni.ubc.ca (G. Bahreini)

\section{To cite this article:}

Mehri Eshaghi, Gholamreza Bahreini. Review on the Role of Flexibility in Iran's Traditional Housing. Case Study: H. H. Ghafouri's (Ghazviniha) Building. International Journal of Science, Technology and Society. Special Issue: Research and Practice in Architecture and Urban Studies in Developing Countries. Vol. 3, No. 2-1, 2015, pp. 138-142. doi: 10.11648/j.ijsts.s.2015030201.36

\begin{abstract}
In Iranian traditional architecture, flexibility as a general concept has always been with the major concerns to from the spatiotemporal aspects of the design. Considering limitations and availability of resources, this area still suffers from lack of brilliant and excellent research to link these positive aspect of traditional systems with those demands and dynamic nature of the modern architectures. Flexibility in general is highly dependent on a variety of factors including socioeconomic and cultural aspect of the society and single families which result in a continuous challenge in its adaptability with the design systems for a long time. In this paper, a review on flexible design of traditional housing is made followed by a descriptive study on one of the famous Iranian traditional houses as a case study, with a focus on the potentials and of such as system to be use in modern societies. Results show the capability of traditional flexibility patterns to be adapted with dynamic requirements of new architectural designs.
\end{abstract}

Keywords: Flexibility Architecture, Traditional Housing, Adaptability, Sustainability

\section{Introduction}

Flexibility a concept in architectural and housing design, has always been an important and basic element in traditional architectures particularly in housing and residential design. However nowadays with the increasing rate of limited space in one hand, and decreasing tendency for static and fixed operation of different structures on the other hand as well as side effects related to indirect time-cost interfere with the design, flexibility has even become more popular during the recent decades. Flexibility in architecture backs to the ancient times and early civilizations, however there are many evidences that academic-based design of flexible housing was mainly contributed by traditional architecture of old civilizations including Iranian architectural and housing relics. People usually pay attention to what they use and where they live and whether those could meet their needs for current and future requirements, considering possible changes in their status and consequences as well as this concern has always been taken into account by flexible housing design in traditional architecture. Community as well as daily requirements of target societies and families. Flexibility in Iranian architectural history, could easily be distinguished in many traditional housing as a basic element of design. However this potential rarely has been applied to the modern architectures despite the growing demands for it by end-users. It is worthy to mention that a redefinition of the concept and its application as design as whet modern architecture is in need to be able to benefit from all the positive aspects of flexibility in design in different fields. Industrialization and development of urbanized regions anywhere in the world, together with highly intensification of time and cost factors in design, has undoubtedly increased the need for flexible housing to be considered as a main tool to fulfill the condition and meet the future demands of the diverse community as well.

\section{Concept of Flexibility}

Flexibility literally means the merit of capability to adopt with any situation as well as environment and is typically referred to the changeability in the system that achieves the terms and conditions and the potential of accountability for the 
future needs [1].

Flexibility is an approach in design which respond to the customers' needs with probable changes in the project pattern consciously or non- consciously. Also it provides a flexible design to variable wills by looking to its application [2]. Many authors focused on this issue and reviewed different aspects of flexibility in design ([4] [14]).

\section{Flexibility in Iranian Traditional Houses}

Flexibility has long been used as a desirable quality in urban and rural traditional housing in Iran. The traditional architecture's flexibility has always been one of the key concepts and basic parts of social and spatial elements. It includes adaptability, transformability, and variability.

Adaptability is probably the more popular form of flexibility that mainly focusses on changeability in nature of architecture to response to changes related to dynamic needs and conditions. Concept of multi-functional spaces is a basic part of flexibility which refers to transformability and could be fit into different concepts and scales, from changing the performances of the components themselves or their multi-functions or form the change in physical spaces and shapes of rooms or other inside and outside spaces. The concept of variability mainly focusses on the change in physical spaces with seasonal and daily changes includes many aspects of architectural design. Generally, variability mainly applies to the large-scale concept and uses different sections in variable conditions. In each of three defined types, time plays an important role. Considering the role of temporal variability, leads to more design complexities. Adaptability and convertibility in locations and spaces within the home are sometime a function of changing in day time or seasonal effects. For instance, using the roof in spring season and sleeping outdoors in summer nights or using the porches in the summer morning and settling hall in the summer noon are all examples of adaptability to change in seasonal time. Variability, transformability and convertibility of the main spaces of traditional houses have always been depended on more important factors of the families such as family development and growth as well as its evolution through the time which usually happens in a larger scale.

A good example of described Iranian`s traditional flexible architecture usually considers several multifunctional spatial elements and concepts. This feature provides the inhabitants with the potential to change the spaces to meet their needs anytime.

This is with no doubt to express that the house of Haj Hassan Ghafouri located in old city of Isfahan is one of the famous architectures in this category. Its history backs to Safavi Era and its antiquity dates back to Nasser Al Din Shah Qajar one of the Iranian most famous kings in that time. In this article, a variety of flexibility concepts have been addressed in addition to reviewing and investigating the case study.

\subsection{Variability (Multi-functional Spaces)}

One of the most noticeable aspects of flexibility in Iranian traditional houses is their multi-functionality performance. Variability provides the ability to use the space in different ways which depends on the spatiotemporal characteristics of the operations and functionality of the building. By definition, any space can be used in different ways for several purposes in predefined period of time or even for different functions in a certain time as well.

At traditional homes the variability becomes a feasible tool as far as it follows a widely accepted pattern and fulfills the standard basic order known as "measure". Due to the location of the yard at the center of most traditional structures, it acts usually as a divider of accessible functions and by itself is also capable of being converted into a public space for the ceremonies and parties as well. This application of centrality is a functional and conceptual element rather than a physical concern. Depth of bright spaces vary in different yard positions. Sometimes the depth is as high as the vault of the compassed walls and sometimes the same as hall room and windward.

Natural access to the rooms through yard and connecting aisles (Capital) provides their functional diversity. This way of internal connections lead to a combination of internal spaces and reduce the wasting of space for connecting corridors. In this viewpoint even connecting units as the capital are a connector of two adjacent rooms which also play as a multi-functional element by themselves. Variability in the service area and main units of the home in first layer surrounding the yard is even more multi-functional than connectors and access providing structures. Different functions and diverse performance of these spaces were provided by a variety of tools such as three-door, five-door rooms and so tailored to be consonant with the daily needs of the families. Each space was created usually in a different period of time. Three- door room option was usually used for settling the family privately doing the works such as weaving the carpet and sleep purposes. The most constant parts of traditional houses were those serving the spaces such as kitchen where located usually far farther from the courtyard. (Figure 1).

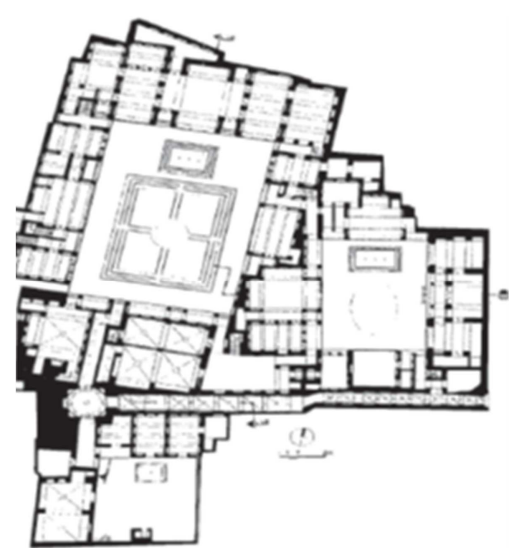

Figure 1. Ground floor plan of case study: Haj Hassan Ghafouri (Qazviniha) -H.H.G. house [3]. 
The elements such as entrance corridor or vestibule in addition to their primary performance as to create the connection between outside and inside spaces and linking public/semipublic space to the private one, also act independently to prepare a divider area as well as make the adjacent neighbors connected. Entrance corridor is sometimes too long in many cases while a bit shorter in other ones. Long corridors act as private lounges as well. (Figures 2 and 3).

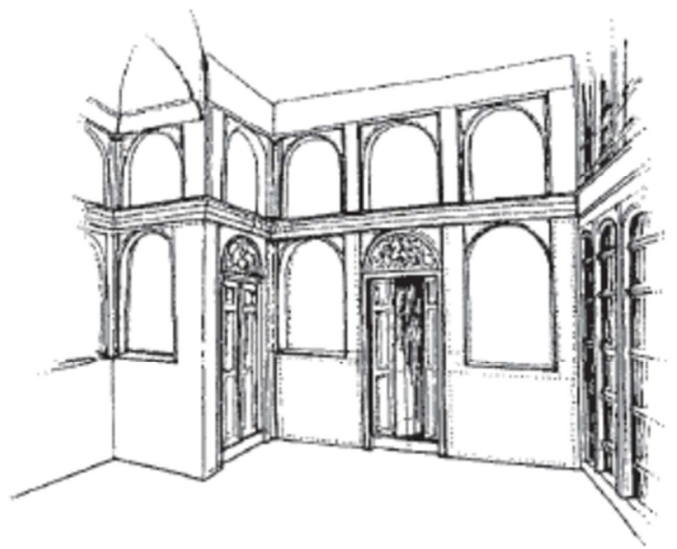

Figure 2. Entrance of main hall of H.H.G. house.

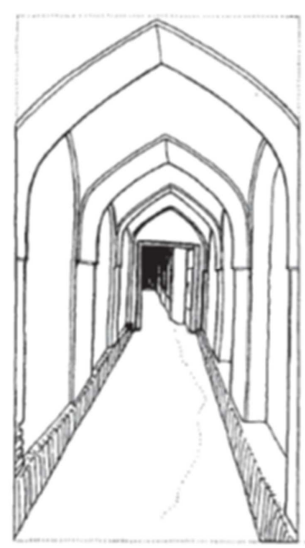

Figure 3. Entrance corridor of H.H.G. house.

There are also a variety of elements forming variability in smaller scale in houses. For instance, the facade at the entrance to the house with hierarchical concept provides also a sitting space (settling space) and there are other spaces with similar functions as well. There are numerous constituent elements as different spaces such as rooms with several layers of wall that provide this capability in the house. The elements such as niches and shelves decrease the thickness of structural walls by lightening the building weight in one hand and act as place to hold stuffs. On the other hand. Many of these objects were also used that time as ornamental signs for luxury houses. (Figure 4).

Other elements such as windows and doors unlike their present specific functions have also been used to serve as multi-function elements serving to connect the yard, room sand gates, lightening the internal corridors and spaces and similar functions at the same time.

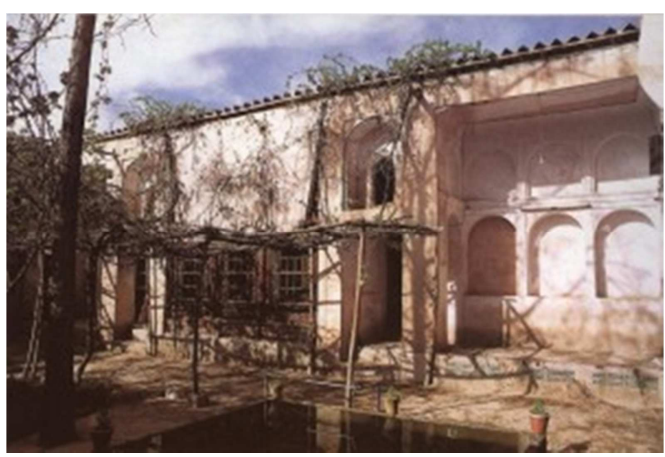

Figure 4. Multi-functional space of yard in H.H.G. house.

Although Sash type of windows rarely were used to pass by, they contribute to make a diverse space inside the house by using colorful glasses affected by changing the natural light source during the day and night times (Figure 5).

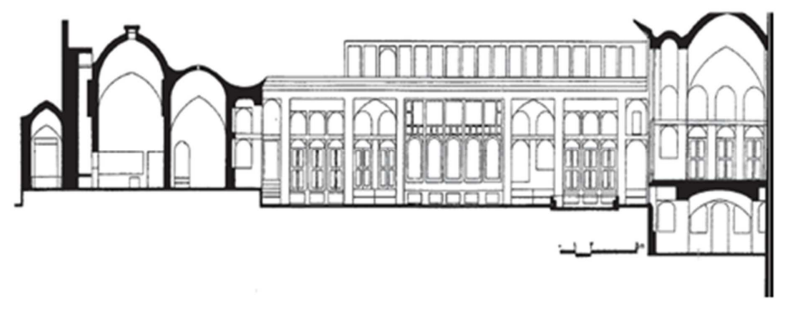

Figure 5. Elevation and eastern view of internal courtyard-H.H.G. house.

\subsection{Adaptability}

Along with daily and seasonal changes, different parts of the house were adopted by the required functions in traditional architectures. Yard act as a tool to make the other spaces with various functions and setting all connected and linked together at the same time. The elements of the house yard has an important role in regulating these functions. For instance, placing the dock in the center of yard and using it in good weather was quite common. Using a wooden flat bench to sit in summer as well as growing appropriate kinds of plants in different seasons was just an example of courtyard multi-function to adapt with the residents' needs. In our case, some facilities such as wooden flats are the elements that facilitate adapting the yard space with the variable needs in summer times.

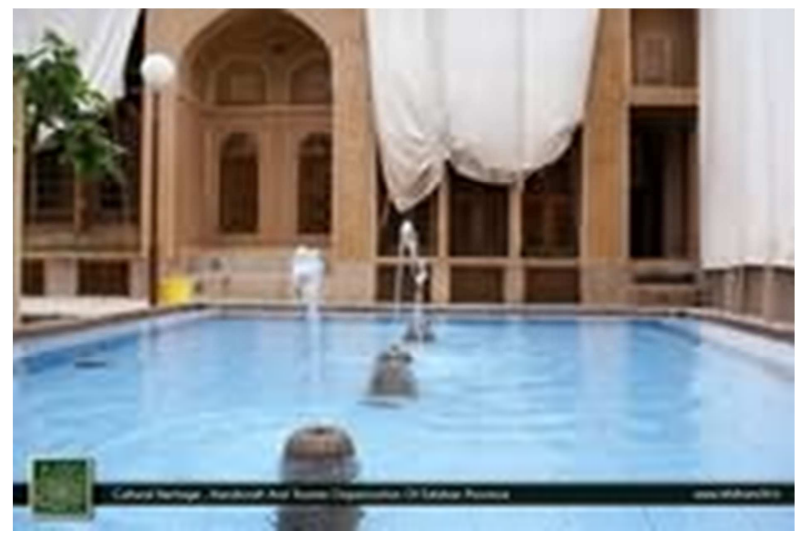

Figure 6. Yard view of H.H.G house. 
Separation of living spaces for summer and winter times in accordance with the change in needs of residents, plays an important role in internal design of the house. Usually the back to sun front of the house was mainly used during summers with a higher wall and hence creating more shadow while the opposite side was used mainly during winter times. Usually an open and depth porch was placed at the edge of summer sitting area. There are also rooms in western front of the yard which used the east light and usually have little depth so appropriate to be used in all seasons. For the houses that were built in three fronts of the yards usually there were not any room built in east side exposed to the west light. Therefore, although yard is central space of house in function, geometrically was not centrically located but most probably was located in a side of the house.

Accordingly, the basement or upstairs at house facilitates changeability potential in different seasonal and annual conditions. For example, in summer nights, upstairs and spring bedroom were used by the privacy and without offense to the neighbors while dinning, resting and sleeping. Home basement in the noon and evening during summer times also provided such applications. Spaces such as hall, three- door, five-door rooms, etc. had also the capability to adapt with dynamic needs based on seasonally and annually differences. Rooms like hall and five-door spaces were likely to adapt with climate change conditions, adding or eliminating non-structural elements.

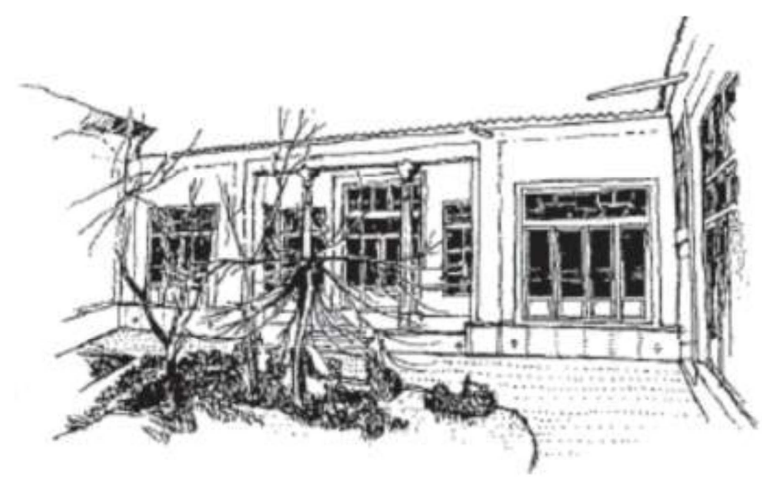

Figure 7. East view of external yard in H.H.G. house.

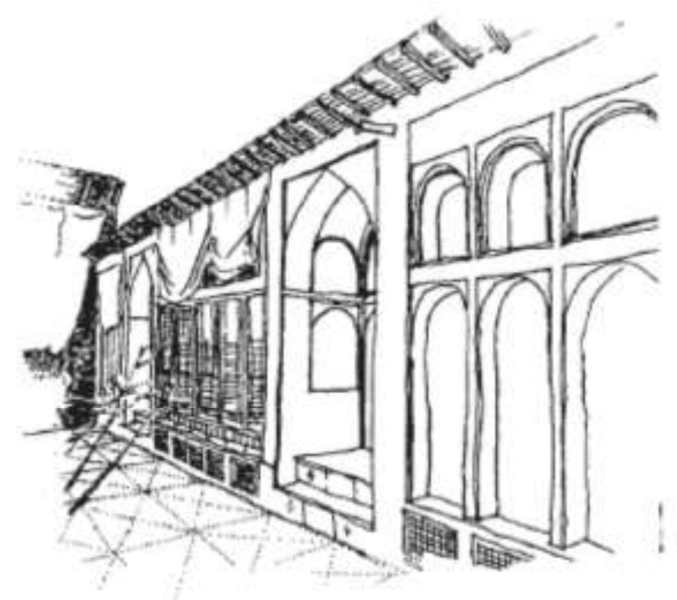

Figure 8. East view of internal yard in H.H.G. house.

\subsection{Convertibility}

Convertibility and transferability of different spaces in traditional houses, have always been considered to deal with variable functions and needs. In many traditional Iranian houses based on change in family size and situation and gradually increasing need for more private spaces rather than group places, a separation in multi-functional yards and dividing bigger rooms into smaller living areas started.

In some cases, the family houses shall gradually be divided into separate units or independent housing with a less population to convert to the new form required for new family conditions. In some cases where the accessible yard space were limited the flexibility in this concept resulted in separation of the surrounding central yard. This way of separating has usually been initiated when the young member of the family wanted to establish his/her new family and start life independently

Therefore, convertibility or kinds of changeability have always been part of design and operation in any scale of traditional houses. Traditional building systems seems to be responsive sufficiently to the needs of residences by the use of large and small "Measures". These spatial changes could be also observed on the smaller scales of external yards and much more significant in different parts of internal yards. It should also be noticed that, the service yard of the houses often were smaller than the main yards and only were built in accordance with the residents' needs, again a factor of flexibility in overall design. Due to relatively small size of service yards to that of internal and external ones, mainly because of mentioned fact and also providing an effective connection between internal and external yards, this was a one story element that also provided livestock with access to the stable and warehouse as well.

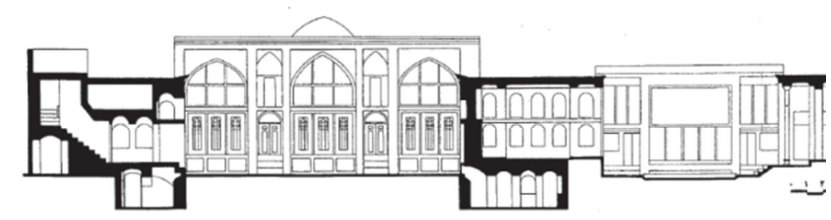

Figure 9. Section and northern view of the external and internal yards in H.H.G house.

\section{Conclusion and Remarks}

Flexibility as a concept has been extensively utilized and designed in Iranian traditional architectures. What has been mostly been considered as the major principle of flexibility in Iranian-Islamic traditional architecture flexibility as a concept rather than physical flexibility. Although, the flexibility might be summarized in any aspects of final structure, there are many consequences of such a concept in social, economic and cultural life of the residents that might affect the concept and how to adjust that in real systems. Following items could be obtained from a review on the case studies in Iranian traditional architectures with a focus on flexibility aspects of their design: 
Generally in traditional houses, unlike modern buildings the name of spaces is a kind of representative of their functions. This means that in Iranian traditional houses rarely there were spaces called as bedroom, living or dining rooms, most of all they were named as concepts such as three-door, five-door and alcove room [5]. This indicates that all these spaces have always been taken into account as flexible elements. For example, a room could be operated as the living room during the day time and be used as a bedroom during the nights. It could be imagined that, using fixed furniture is one of the reasons limiting the flexibility potential in modern houses that prevents the space to serve for different functions while this concept have been used effectively in Iranian traditional houses using an appropriate decorations and utilizing flexible design.

In traditional houses, main building blocks were usually defined in two north and south directions. North as mainly a preferred living and working area during summer and south as sitting in winter times. Family often moved and stayed in winter area and reside there. They spent day time mainly on the porch and in internal parts during nights. In summer times however, living continues in the southern part, bed chambers and cellars were solutions to extreme heat for family members while they also sometimes preferred to stay just by the pond as the air conditioning was quite pleasant especially during the summer nights.

In traditional houses the roof was also a place for local ceremonies during nights and a place to sleep as well. This kind of movement and spatial definitions indicate different responses to variable needs of residents. Although this type of flexibility might not be feasible in modern buildings due to the limited space especially in urban areas, however using natural conditions as energy sources could be applied also in our modern architecture as energy saving approach in design, the area which traditional houses tried to use that possibility as much as possible and we have to follow those simple rules considering limited fossil-based sources of energy.

In traditional houses the spaces have been used in a multi-functional flexible way. Roof, porch, yard and other spaces were all designed and built in a way to serve as a multi-function element. This finally could build an efficient system to meet the current and future needs of the families by providing them a flexible choices of spaces that could adapt with dynamic and variable status and requirements.

There is no doubt that new modern design could not follow the same rules as existed before, however looking at traditional architecture patterns and methods reveals a variety of techniques used to better response to the resident's needs. Among those, flexibility is a general concept that found to be capable to deal with many dynamic and variable needs of a specific architecture. In Iranian Islamic-Traditional architecture, however many cases were found to have the flexibility as a concept in design. Haj Hassan Ghafouri (Qazviniha) house is one of the most famous monuments that represents many aspects of flexibility in design, build and operation of a residential architecture.

\section{References}

[1] Moein, Muhammad, Persian culture, 1st volume, published by Amir-Kabir, 8th Edition, Tehran, 1992.

[2] Habibi, Masoud, Designing functional diagram structure in residential homes, Case study of designing flexible plan, Regeonal Conferrence on Iranian Housing, Gonbad Kavoos, 2010 .

[3] Treasure letters, Islamic Architecture Culture, Isfahan Homes, Center of Documents and Research, School of Architecture and Urbanization, 1999.

[4] Eynifar, Alireza, "A model to Analyze Flexibility of Traditional Housing in Iran, Fine Arts, No. 13, 2013.

[5] Pirnia, Karimi, Iranian House discussed by Karim Pirnia, Abadi Journal, 6.23, 1996.

[6] Zandyeh, Mehdi, Eghbali, Rahman, Hesari, Pedram, Design Methods of Flexible Architectures, Naghshe Jahan Science and Research Journal, 2011.

[7] Pena, William M., and Steven A. Parshall. Problem seeking: An architectural programming primer. John Wiley \& Sons, 2012.

[8] Zeidler, Eberhard H. Multi-use architecture in the urban context. Van Nostrand Reinhold Company, 1985.

[9] Altaş, Nur Esin, and Ahsen Özsoy. "Spatial adaptability and flexibility as parameters of user satisfaction for quality housing." Building and environment, 33.5, pp. 315-323, 1998.

[10] Schneider, Tatjana, and Jeremy Till. Flexible housing. Architectural press, 2007.

[11] Till, Jeremy, and Tatjana Schneider. "Flexible housing: the means to the end." Architectural Research Quarterly 9.3-4, pp. 287-296, 2005.

[12] John Habraken, N. "Design for flexibility." Building Research \& Information 36.3, pp. 290-296, 2008.

[13] Horne, Lee. Village spaces: settlement and society in northeastern Iran. Smithsonian Institution Press, 1994.

[14] Shabani, M., et al. "Achieving Privacy in the Iranian Contemporary Compact Apartment Through Flexible Design." Selected Topics in Power System and Remote Sensing, 2010. 